\title{
Reinforcing a Matroid to Have $k$ Disjoint Bases
}

\author{
Hong-Jian Lai ${ }^{1,2}$, Ping $\mathrm{Li}^{2}$, Yanting Liang ${ }^{2}$, Jinquan $\mathrm{Xu}^{3}$ \\ ${ }^{1}$ College of Mathematics and System Sciences, Xinjiang University, Urumqi, China \\ ${ }^{2}$ Department of Mathematics, West Virginia University, Morgantown, USA \\ ${ }^{3}$ Department of Mathematics, Huizhou University, Huizhou, China \\ E-mail:hjlai@math.wvu.edu \\ Received May 14, 2010; revised July 29, 2010; accepted August 2, 2010
}

\begin{abstract}
Let $\tau(M)$ denote the maximum number of disjoint bases in a matroid $M$. For a connected graph $G$, let $\tau(G)=\tau(M(G))$, where $M(G)$ is the cycle matroid of $G$. The well-known spanning tree packing theorem of Nash-Williams and Tutte characterizes graphs $G$ with $\tau(G) \geq k$. Edmonds generalizes this theorem to matroids. In [1] and [2], for a matroid $M$ with $\tau(M) \geq k$, elements $e \in E(M)$ with the property that $\tau(M-e) \geq k$ have been characterized in terms of matroid invariants such as strength and $\eta$-partitions. In this paper, we consider matroids $M$ with $\tau(M)<k$, and determine the minimum of $\left|E\left(M^{\prime}\right)\right|-|E(M)|$, where $M^{\prime}$ is a matroid that contains $M$ as a restriction with both $r\left(M^{\prime}\right)=r(M)$ and $\tau\left(M^{\prime}\right) \geq k$. This minimum is expressed as a function of certain invariants of $M$, as well as a min-max formula. These are applied to imply former results of Haas [3] and of Liu et al. [4].
\end{abstract}

Keywords: Disjoint Bases, Edge-Disjoint Spanning Trees, Spanning Tree Packing Numbers, Strength, Fractional Arboricity

\section{Introduction}

In this paper, we use $N$ and $Q_{+}$to denote the set of all natural numbers and the set of all positive fractional numbers, respectively, and consider finite matroids and graphs. Undefined notations and terminology can be found in [5] or [6] for matroids, and [7] for graphs. Thus for a connected graph $G, \omega(G)$ denotes the number of components of $G$. For a matroid $M, r_{M}$ (or $r$, when the matroid $M$ is understood from the context) denotes the rank function of $M$, and $E(M), I(M)$, $C(M)$ and $B(M)$ denote the ground set of $M$, and the collections of independent sets, the circuits, and the bases of $M$, respectively. Furthermore, if $M$ is a matroid with $E=E(M)$, and if $X \subset E$, then $M-X$ is the restricted matroid of $M$ obtained by deleting the elements in $X$ from $M$, and $M / X$ is the matroid obtained by contracting elements in $X$ from $M$. As in [5] or [6], we use $M-e$ for $M-\{e\}$ and $M / e$ for $M /\{e\}$.

For a matroid $M$, let $\tau(M)$ denote the maximum number of disjoint bases of $M$. For a graph $G$, define $\tau(G)=\tau(M(G))$, where $M(G)$ denotes the cycle matroid of $G$. Thus if $G$ is a connected graph, then $\tau(G)$ is the spanning tree packing number of $G$.
Readers are referred to [8] for a survey on $\tau(G)$. The well-known spanning tree packing theorem of NashWilliams [9] and Tutte [10] characterizes graphs with $k$ edge-disjoint spanning trees, for any integer $k>0$. Edmonds [11] proved the corresponding theorem for matroids.

Let $k>0$ be an integer. For any matroid $M$ with $\tau(M) \geq k$, we are interested in finding elements $e \in E(M)$ that have the property that $\tau(M-e) \geq k$. Characterizations of all such elements have been found in [1] and [2]. For a graph $G$, the problem of determining which edges should be added to $G$ so that the resulting graph has $k$ edge-disjoint spanning trees has been studied, see Haas [3] and Liu et al. [4], among others. As the arguments in these papers are involved vertices, it is natural to consider the possibility of extending these results to matroids. Since matroids in general do not have a concept corresponding to vertices, one can no longer add an element to a matroid as adding an edge in graphs. Therefore, we need to reformulate the problem so that it would fit the matroid setting while generalizing the graph theory results.

Let $M$ be a matroid and $k \in N$. If there is a matroid $M^{\prime}$ with $r\left(M^{\prime}\right)=r(M)$ and $\tau\left(M^{\prime}\right) \geq k$ such that $M^{\prime}$ has a restriction isomorphic to $M$ (we then 
view $M$ as a restriction of $M^{\prime}$ ), then $M^{\prime}$ is a $(\tau \geq k)$-extension of $M$. We shall show that any matroid has a $(\tau \geq k)$-extension. We then define $F(M, k)$ to be the minimum integer $l>0$ such that $M$ has a $(\tau \geq k)$-extension $M^{\prime}$ with $\left|E\left(M^{\prime}\right)\right|-|E(M)|=l$. The main purpose of this paper is to determine $F(M, k)$ in terms of other invariants of $M$.

By definition, if $M$ is a matroid with $r(M)=0$, then $\forall k \in N, \tau(M) \geq k$. Accordingly, for a connected graph $G$, if $|V(G)|=1$, then $\tau(G) \geq k$ for any $k \in N$. For a graph $G, a_{1}(G)$, the edge arboricity of $G$, is the minimum number of spanning trees of $G$ whose union equals $E(G)$. For a matroid, we define the similar concept $\gamma_{1}(M)$, which is the minimum number of bases of $M$ whose union equals $E(M)$. The following theorems are well known.

Theorem 1.1 Let $G$ be a connected graph with $|V(G)|>1$, and let $k>0$ be an integer. Each of the following holds.

1) (Nash-Williams [9] and Tutte [10]) $\tau(G) \geq k$ if and only if $\forall X \subseteq E(G),|X| \geq k(\omega(G-X)-1)$.

2) (Nash-Williams [12]) $a_{1}(G) \leq k$ if and only if $\forall X \subseteq E(G),|X| \leq k(|V(G[X])|-\omega(G[X]))$.

Theorem 1.2 (Edmonds [11]) Let $M$ be a matroid with $r(M)>0$. Each of the following holds.

1) $\tau(M) \geq k$ if and only if $\forall X \subseteq E(M)$, $|E(M)-X| \geq k(r(M)-r(X))$.

2) $\gamma_{1}(M) \leq k$ if and only if $\forall X \subseteq E(M)$, $|X| \leq k r(X)$.

Let $M$ be a matroid with rank function $r$. For any subset $X \subseteq E(M)$ with $r(X)>0$, the density of $X$ is

$$
d_{M}(X)=\frac{|X|}{r_{M}(X)} .
$$

When the matroid $M$ is understood from the context, we often omit the subscript $M$. We also use $d(M)$ for $d(E(M))$. Following [13] and [14], the strength $\eta(M)$ and the fractional arboricity $\gamma(M)$ of $M$ are respectively defined as

$$
\begin{aligned}
& \eta(M)=\min \{d(M / X): r(X)<r(M)\}, \\
& \text { and } \gamma(M)=\max \{d(X): r(X)>0\} .
\end{aligned}
$$

Thus Theorem 1.2 above indicates that

$$
\tau(M)=\lfloor\eta(M)\rfloor \text {, and } \gamma_{1}(M)=\lceil\gamma(M)\rceil .
$$

We assume that $M$ is a matroid with $r(M)>0$. A subset $X \subseteq E(M)$ is an $\eta$-maximal subset and $M \mid X$ is an $\eta$-maximal restriction if for any subset $Y \subseteq E(M)$ that properly contains $X$, we have $\eta(M \mid Y)<\eta(M \mid X)$. In [1] and [2], it has been proved that any matroid $M$ has a unique decomposition based on its $\eta$-maximal subsets.

Theorem 1.3 ([1] and [2]) Let $M$ be a matroid with $r(M)>0$. Then each of the following holds.

1) There exist an integer $m \in N$, and an $m$-tuple $\left(l_{1}, l_{2}, \ldots, l_{m}\right)$ of rational numbers in $Q_{+}$such that

$$
\eta(M)=l_{1}<l_{2}<\ldots<l_{m}=\gamma(G),
$$

and a sequence of subsets

$$
J_{m} \subset \ldots \subset J_{2} \subset J_{1}=E(M) ;
$$

such that for each $i$ with $1 \leq i \leq m, M \mid J_{i}$ is an $\eta$ maximal restriction of $M$ with $\eta\left(M \mid J_{i}\right)=l_{i}$.

2) The integer $m$ and the sequences (4) and (3) are uniquely determined by $M$.

For a matroid $M$, the $m$-tuple $\left(l_{1}, l_{2}, \ldots, l_{m}\right)$ and the sequence in (4) will be referred as the $\eta$-spectrum and the $\eta$-decomposition of $M$, respectively. For each subscript $j$ with $1 \leq j \leq m$, we refer $J_{j}$ to be the set corresponding to $l_{j}$. Our main result can now be stated as follows.

Theorem 1.4 For $k \in N$, let $M$ be a matroid with $\tau(M) \leq k$. If $\gamma(M)<k$, define $J_{i(k)}=\varnothing$; and if $\gamma(M) \geq k$, let $i(k)$ denote the smallest subscript in (3) such that $l_{i(k)} \geq k$. Then

1) $F(M, k)=k\left(r(M)-r\left(J_{i(k)}\right)\right)-\left|E(M)-J_{i(k)}\right|$.

2) $F(M, k)=\max _{X \subseteq E(M)}\{k r(M / X)-|M / X|\}$.

In the next section, we shall present some of the useful properties related to the strength and the fractional arboricity of a matroid $M$, and to the decomposition of $M$. Section 3 will be devoted to the proofs of the main results. In the last section, we shall show some applications of our main results.

\section{Preliminaries}

Both $\eta(M)$ and $\gamma(M)$ have been studied by many, see [14-16] and [17], among others. From the definition of $d(M), \eta(M)$ and $\gamma(M)$, we immediately have, for any matroid $M$ with $r(M)>0$,

$$
\eta(M) \leq d(M) \leq \gamma(M) .
$$

A matroid $M$ satisfying $\eta(M)=\gamma(M)$ is called a uniformly dense matroid. Both $\eta(M)$ and $\gamma(M)$ can also be described by their behavior in some parallel extension of the matroid \$M\$.

Definition 2.1 Let $M$ be a matroid and let $\phi: E(M) \mapsto N$ be a function. For each $e \in E(M)$, let $X_{e}=\left\{e^{1}, e^{2}, \cdots, e^{\varphi(e)}\right\}$ be a set such that $X_{e} \cap X_{e^{\prime}}=\varnothing$, $\forall e, e^{\prime} \in E(M)$ with $e \neq e^{\prime}$. The $\phi$-parallel extension of $M$, denoted by $M_{\phi}$, is obtained from $M$ by replacing each element $e \in E(M)$ by a class of $\phi(e)$ parallel elements $X_{e}$. Thus $E\left(M_{\varphi}\right)=\bigcup_{e \in E(M)} X_{e}$ such that a subset $Y \subseteq E\left(M_{\phi}\right)$ is independent in $M_{\phi}$ if and 
only if both $\left\{e \in E(M): X_{e} \cap Y \neq \varnothing\right\}$ is independent in $M$ and $\forall e \in E(M),\left|X_{e} \cap Y\right| \leq 1$. For $t \in N$, if $\forall e \in E(M), \phi(e)=t$ is a constant function, we write $M_{t}$ for $M_{\phi}$, and call $M_{t}$ the $t$-parallel extension of $M$.

Let $E^{\prime}=\left\{e^{1}: e \in E(M)\right\} \subseteq E\left(M_{\varphi}\right)$. Then the bijection $e \leftrightarrow e^{1}$ between $E(M)$ and $E^{\prime}$ yields a matroid isomorphism between $M$ and $M_{\phi} \mid E^{\prime}$. Under this bijection, we shall view that $M=M_{\phi} \mid E^{\prime}$ is a restriction of $M_{\phi}$.

Theorem 2.2 (Theorem 4 of [14]) Let $M$ be a matroid and let $s \geq t>0$ be integers. Then

1) $\eta(M) \geq \frac{s}{t}$ if and only if $\eta\left(M_{t}\right) \geq s$.

2) $\gamma(G) \leq \frac{s}{t}$ if and only if $\gamma\left(M_{t}\right) \leq s$.

Theorem 2.3 (Theorem 6 of [14]) Let $M$ be a matroid. The following are equivalent.

1) $\eta(M)=d(M)$.

2) $\gamma(M)=d(M)$.

3) $\eta(M)=\gamma(M)$.

4) $\eta(M)=\frac{s}{t}$, for some integers $s \geq t>0$, and $M_{t}$, the $t$-parallel extension of $M$, is a disjoint union of $s$ bases of $M$.

5) $\gamma(M)=\frac{s}{t}$, for some integers $s \geq t>0$, and $M_{t}$, the $t$-parallel extension of $M$, is a disjoint union of $s$ bases of $M$.

Lemma 2.4 ([14], [1] and [2]) Let $M$ be a matroid with $r(M)>0$, and let $l \geq 1$ be fractional number. Each of the following holds.

1) (Lemma 10 [14]) If $X \subset E(M)$ and if $\eta(M \mid X) \geq \eta(M)$, then $\eta(M / X)=\eta(M)$.

2) (Theorem 17 of [14]) If $X \subset E(M)$ and if $d(X)=\gamma(M)$, then $\eta(M \mid X)=\gamma(M \mid X)=d(X)=\gamma(M)$.

3) A matroid $M$ is uniformly dense if and only if any subset $X \subseteq E(M), d(X) \leq \eta(M)$.

4) A matroid $M$ is uniformly dense if and only if for any restriction $N$ of $M, \eta(N) \leq \eta(M)$.

5) If $d(M) \geq l$, then there exists a subset $X \subseteq E(M)$ with $r(X)>0$ such that $\eta(M \mid X) \geq l$.

For each rational number $l>1$, define

$$
S_{l}=\{M: \eta(M) \geq l\} .
$$

Proposition 2.5 ([1] and [2]) Let $p>q>0$ be integers, and $l=\frac{p}{q} \in Q_{+}$be a rational number. The matroid family $S_{l}$ satisfies the following properties.

(C1) If $r(M)=0$, then $M \in S_{l}$.
(C2) If $M \in S_{l}$ and if $e \in E(M)$, then $M / e \in S_{l}$.

(C3) Let $X \subseteq E(M)$ and let $N=M \mid X$. If $M / X \in S_{l}$ and if $N \in S_{l}$, then $M \in S_{l}$.

Lemma 2.6 ([1] and [2]) Let $W, W^{\prime} \subset E(M)$ be subsets, and let $l \in Q_{+}$. If $\eta(M \mid W) \geq l$ and $\eta\left(M \mid W^{\prime}\right) \geq l$, then $\eta\left(M \mid\left(W \cup W^{\prime}\right)\right) \geq l$.

Lemma 2.7 ([1] and [2]) If $X \subseteq E(M)$ is an $\eta$ maximal subset, then $X$ is a closed set in $M$.

\section{Characterization of the Must-Added Elements with Respect to Having $k$ Disjoint Bases}

The main purpose of this section is to prove Theorems 1.4. We will start with a lemma.

Lemma 3.1 Let $M$ be a matroid and let $k>0$ be an integer. Each of the following holds.

1) $\eta(M) \geq k$ if and only if $F(M, k)=0$.

2) If $\gamma(M) \leq k$, then

$$
F(M, k)=k r(M)-|E(M)| .
$$

Moreover, there exists a map $\phi: E(M) \mapsto N$, such that $M_{\phi}$ is a matroid that contains $M$ as a restricttion with $\eta\left(M_{\phi}\right)=\gamma\left(M_{\phi}\right)=k$, and such that $\left|E\left(M_{\varphi}\right)\right|-|E(M)|=F(M, k)$.

Proof: 1) By (2), $\eta(M) \geq k$ if and only if $\tau(M) \geq k$. By the definition of $F(M, k), \tau(M) \geq k$ if and only if $F(M, k)=0$. This proves 1$)$.

2) Since $\gamma(M) \leq k$, it follows by (2) that $M$ has disjoint bases $B_{1}, \cdots, B_{k}$ such that $E(M)=\bigcup_{i=1}^{k} B_{i}$. Define $\phi(e)=\left|\left\{B_{i}: e \in B_{i}\right\}\right|$. Then $\phi: E(M) \mapsto N$. Let $L=M_{\phi}$ be the $\phi$-parallel extension of $M$. Then by Definition 2.1, $M$ is contained in $L$ as a restriction. Moreover, both $|E(L)|=\sum_{i=1}^{k}\left|B_{i}\right|=k r(M)$ and $\tau(L)=k$. It follows by Theorem 2.3 that $\eta(L)=\gamma(L)=k$. Hence by Theorem 2.31 ) or 2), $|E(L)|=k r(L)=k r(M)$, and so $F(M, k)=|E(L)|-|E(M)|=k r(M)-|E(M)|$.

When $k=2$, the cycle matroid version of Lemma 3.1 has been frequently applied in the study of supereulerian graphs, see Theorem 7 of [18] and Lemma 2.3 of [19], among others. (For a literature review on supereulerian graphs, see [20] and [21].)

Proof of Theorem 1.4 1): Let $M$ be a matroid with $r(M)>0$. If $\tau(M) \geq k$, then by (2) and by Theorem $1.3, i(k)=i_{1}$, and so

$$
E(M)=J_{i(k)} \text {, and } F(M, k)=0 .
$$

Thus Theorem 1.4 1) follows trivially with $\tau(M) \geq k$. Hence we assume that $\tau(M)<k$. If $\gamma(M)<k$, then Theorem 1.4 1) follows from Lemma 3.1.

Therefore, we may assume that $\eta(M)<k$ and $\gamma(M) \geq k$. By Theorem 1.3, we must have $m>1$. Let 
$i(k)$ be the smallest subscript in $\eta$-spectrum (3) of $M$ such that $l_{i(k)} \geq k$. By Theorem 1.3, $\eta\left(M \mid J_{i(k)}\right) \geq k$. Let $M^{\prime}=M / J_{i(k)}$. By the assumption that $\eta(M)<k$ and by Lemma 2.41$), \eta\left(M^{\prime}\right)=\eta(M)$. By the choice of $i(k), \gamma\left(M^{\prime}\right)<k$, and so by Lemma 3.1,

$$
F\left(M^{\prime}, k\right)=k r\left(M^{\prime}\right)-\left|E\left(M^{\prime}\right)\right|,
$$

and there must be a function $\phi^{\prime}: E\left(M^{\prime}\right) \mapsto N$ such that $M_{\phi^{\prime}}^{\prime} \quad$ satisfies $\quad \eta\left(M_{\phi^{\prime}}^{\prime}\right)=\gamma\left(M_{\phi^{\prime}}^{\prime}\right)=k$. Define $\phi$ : $E(M) \mapsto N$ as follows:

$$
\phi(e)=\left\{\begin{array}{ll}
\phi^{\prime}(e) & \text { if } e \notin J_{i(k)} \\
1 & \text { if } e \in J_{i(k)}
\end{array} .\right.
$$

Then $M_{\phi}$ is a matroid that contains $M$ as a restriction, such that $J_{k}(M) \subset E\left(M_{\phi}\right)$. By the definition of $\phi$, $M_{\phi}\left|J_{i(k)}=M\right| J_{i(k)} \in S_{k}$. Since $M_{\phi} / J_{i(k)}=M_{\phi^{\prime}}^{\prime} \in S_{k}$, it follows by Proposition 2.5(C3) that $M_{\phi} \in S_{k}$. Thus by (7) and by Lemma 2.7 ,

$$
\begin{aligned}
& F(M, k)=F\left(M^{\prime}, k\right)=k r\left(M^{\prime}\right)-\left|E\left(M^{\prime}\right)\right| \\
& =k\left(r(M)-r\left(J_{i(k)}\right)\right)-\left|E(M)-J_{i(k)}\right|,
\end{aligned}
$$

and so Theorem 1.4 1) is established.

To continue our proof for Theorem 1.4, we introduce the following function: for any $X \subseteq E(M)$, define

$$
\begin{aligned}
& f_{k}(M, X)=k r(M / X)-|M / X|, \\
& \text { and } F_{k}(M)=\max _{X \subseteq E(M)}\left\{f_{k}(M, X)\right\} .
\end{aligned}
$$

The function $f_{k}(M, X)$ was introduced by Bruno and Weinberg [22] to investigate the principal partition of matroids. They are closely related to the strength and fractional arboricity of matroids, as to be shown in Lemma 3.2 below.

Lemma 3.2 Let $M$ be a matroid with $r(M)>0$, and let $k>0$ be an integer. Each of the following holds.

1) $F_{k}(M)=0$ if and only if $\eta(M) \geq k$.

2) $F_{k}(M)=f_{k}(M, \varnothing)$ if and only if $\gamma(M) \leq k$.

3) Let $i(k)$ denote the smallest $i_{j}$ in (3) such that $i(k) \geq k$, and $J_{i(k)}$ denote the corresponding set in the $\eta$-decomposition (4) of $M$. Then

$F_{k}\left(M / J_{i(k)}\right)=F(M, k)$.

4) For any $e \in E(M), F_{k}(M) \geq F_{k}(M / e)$. In particular, $F_{k}(M) \geq F(M, k)$.

5) If $X_{0} \subset E(M)$ satisfies $F_{k}(M)=f_{k}\left(M, X_{0}\right)$, then $F_{k}(M)=f_{k}\left(M / X_{0}\right)=F_{k}\left(M / X_{0}\right)=f_{k}\left(M / X_{0}, \varnothing\right)$ and $\gamma\left(M / X_{0}\right) \leq k$.

Proof: 1) By definition (8), $F_{k}(M)=0$ if and only if $\forall X \subseteq E(M), \quad f_{k}(M, X)=k r(M / X)-|E(M / X)| \leq 0$. By the definition of $\eta(M), \forall X \subseteq E(M)$, $k r(M / X)-|E(M / X)| \leq 0$ if and only if $\eta(M) \geq k$.

2) By the definition of $F_{k}(M), F_{k}(M)=f_{k}(M, \varnothing)$ if and only if $\forall X \subseteq E(M)$,

$$
k(r(M)-r(X))-|E-X| \leq k r(M)-|E| ;
$$

and so if and only if $\forall X \subseteq E(M)$ with $r(X)>0$, $\frac{|X|}{r(X)} \leq k$. By the definition of $\gamma(M)$, this happens if and only if $\gamma(M) \leq k$.

3) By Theorem 1.3, $\gamma\left(M / J_{i(k)}\right)<k$. By 2) of this lemma, by Lemma 2.7, and by Theorem 1.41 ),

$$
\begin{aligned}
& F_{k}\left(M / J_{i(k)}\right)=f_{k}\left(M / J_{i(k)}, \varnothing\right)=k r\left(M / J_{i(k)}\right)-\left|M / J_{i(k)}\right| \\
& =k\left(r(M)-r\left(J_{i(k)}\right)\right)-\left(|E|-\left|J_{i(k)}\right|\right)=F(M, k) .
\end{aligned}
$$

4) For any $e \in E(M)$, by the definition of $F_{k}(M)$ in (8), $F_{k}(M) \geq F_{k}(M / e)$. It follows by 3$)$ of this lemma that $F_{k}(M) \geq f_{k}(M, X)=F(M, k)$.

5) By 4), and by the choice of $X_{0}$, we have

$$
\begin{aligned}
& F_{k}(M) \geq F_{k}\left(M / X_{0}\right) \geq f_{k}\left(M / X_{0}, \varnothing\right) \\
& =f_{k}\left(M, X_{0}\right)=F_{k}(M) .
\end{aligned}
$$

Thus equalities must hold and so $F_{k}(M)=f_{k}\left(M / X_{0}\right)=F_{k}\left(M / X_{0}\right)=f_{k}\left(M / X_{0}, \varnothing\right) \quad$ It follows by 2) that $\gamma\left(M / X_{0}\right) \leq k$. This proves 5).

Lemma 3.3 Suppose that $X_{0} \subseteq E(M)$ satisfies $f_{k}\left(M, X_{0}\right)=F_{k}(M)$. Then $\eta\left(M \mid X_{0}\right) \geq k$.

Proof: By Lemma 3.11 ), it suffices to show that $F_{k}\left(M \mid X_{0}\right)=0$. For any $Y \subseteq X_{0}$, as

$$
\begin{aligned}
& f_{k}\left(M \mid X_{0}, Y\right)=k\left(r\left(X_{0}\right)-r(Y)\right)-\left|X_{0}\right|+|Y|, \\
& \text { and } f_{k}\left(M, X_{0}\right)=k\left(r(M)-r\left(X_{0}\right)\right)-|E(M)|+\left|X_{0}\right| .
\end{aligned}
$$

It follows that $f_{k}\left(M \mid X_{0}, Y\right)+f_{k}\left(M, X_{0}\right)=f_{k}(M, Y) \leq$ $F_{k}(M)=f_{k}\left(M, X_{0}\right)$. Thus by definition, $f_{k}\left(M \mid X_{0}, Y\right) \leq 0$. This implies that $F_{k}\left(M \mid X_{0}\right)=0$, and so $\eta\left(M \mid X_{0}\right) \geq k$.

Proof of Theorem 1.4 2): By Lemma 3.2 4), it suffices to show that $F_{k}(M) \leq F(M, k)$. We shall argue by induction on $|E(M)|$ to proceed the proof.

Suppose first that $F_{k}(M)=0$. Then by Lemma 3.21 ), $F_{k}(M)=0$ if and only if $\eta(M) \geq k$. By Lemma 3.1 1), we have $F(M, k)=0=F_{k}(M)$ in this case. Thus we assume that $F_{k}(M)>0$.

By Lemma 3.11$), \quad F_{k}(M)>0$ if and only if $\eta(M)<k$. If $\gamma(M) \leq k$, then by Lemma 3.12 ), and by Lemma 3.2 2),

$$
F_{k}(M)=f_{k}(M, \varnothing)=k r(M)-|E(M)|=F(M, k) .
$$

Hence we may assume that Theorem 1.42 ) holds for smaller values of $|E(M)|$, and that

$$
\eta(M)<k<\gamma(M) \text {. }
$$

By induction, we may assume that $M$ does not have loops. By Theorem 1.3, and by (9), both $i(k)$, the smal- 
lest $j$ in (3) such that $l_{j} \geq k$, and $J_{i(k)}$, the corresponding set in (4), exist.

Let $X_{0} \subset E(M)$ be a subset such that $F_{k}(M)=f_{k}\left(M, X_{0}\right)$. By (9), $\quad X_{0} \neq \varnothing$. Since $\left|E\left(M / X_{0}\right)\right|<|E(M)|$, by Lemma 3.25$)$ and by induction, we have

$$
\begin{aligned}
& F_{k}(M)=f_{k}\left(M / X_{0}\right)=F_{k}\left(M / X_{0}\right)=F\left(M / X_{0}, k\right), \\
& \text { and } \gamma\left(M / X_{0}\right) \leq k .
\end{aligned}
$$

Suppose that $F(M, k)=l$. Then there exists a matroid $M^{\prime}$ with $M^{\prime} \in S_{k}$, which contains $M$ as a restriction and satisfies $\left|E\left(M^{\prime}\right)-E(M)\right|=l$. Note that $X_{0} \subseteq E(M) \subseteq E\left(M^{\prime}\right)$. Let $W=E\left(M^{\prime}\right)-E(M)$, and $W_{0}=W-c l_{M^{\prime}}\left(X_{0}\right)$. Then $\left|W_{0}\right| \leq|W|$.

Since $M^{\prime} \in S_{k}$, it follows by Proposition 2.5 (C2) that $M^{\prime} / X_{0} \in S_{k}$. Since $M$ is a restriction of $M^{\prime}$, $M / X_{0}$ is a restriction of $M^{\prime} / X_{0}$. It follows by the definition of $F\left(M / X_{0}, k\right)$ and by (10) that

$$
\begin{aligned}
& F_{k}(M)=F\left(M / X_{0}, k\right) \leq\left|E\left(M^{\prime} / X_{0}\right)-E\left(M / X_{0}\right)\right| \\
& \leq\left|W_{0}\right| \leq|W|=F(M, k) .
\end{aligned}
$$

This, together with Lemma 3.24 ), implies Theorem 1.4 2).

\section{Applications}

Let $G$ be a graph, and $M=M(G)$ be the cycle matroid of $G$. Let $F(G, k)=F(M(G), k)$, and $f_{k}(G, X)=f_{k}(M(G), X)$, for any edge subset $X \subseteq$ $E(G)$. Let $\omega(G)$ denote the number of connected components of $G$. The next theorem follows immediately from Theorem 1.4.

Theorem 4.1 (Theorems 3.4 and 3.10 of [4]) For $k \in N$, let $G$ be a connected graph with $\tau(M(G)) \leq k$ and let $i(k)$ denote the smallest $i_{j}$ in (3) such that $i(k) \geq k$. Then

1) $\quad F(G, k)=k\left(|V(G)|-\left|V\left(G\left[J_{i(k)}\right]\right)\right|+\omega\left(G\left[J_{i(k)}\right]\right)\right.$ $-1)-\left|E(G)-J_{i(k)}\right|$.

2) $F(G, k)=\max _{X \subseteq E(G)}\left\{f_{k}(G, X)\right\}$.

The problem of reinforcing graphs to have $k$ edgedisjoint spanning trees has also been investigated by others. In [3], the following is proved.

Theorem 4.2 (Haas, Theorem 1 of [3]) The following are equivalent for a graph $G$, and integers $k>0$ and $l>0$.

1) $|E(G)|=k(|V(G)|-1)-l$ and for subgraphs $H$ of $G$ with at least 2 vertices, $|E(H)| \leq k(|V(H)|-1)$.

2) There exists some $l$ edges which when added to $G$ result in a graph that can be decomposed into $k$ spanning trees.

Proof: Assume that 1) holds. Then by 1), $\gamma(M(G)) \leq k$. It follows by the assumption that $|E(G)|=k(|V(G)|-1)-l$ and by Lemma 3.12$)$ that
$F(G, k)=l$, and so 1$)$ is obtained.

Assume 2) holds. Since adding $l$ edges to $G$ can result in a graph in $S_{k}$, by (1) and by (2), $\gamma(M(G)) \leq k$. By Lemma 3.12 ),

$$
k(|V(G)|-1)-|E(G)|=F(G, k)=l,
$$

and so 2) must hold.

\section{References}

[1] H.-J. Lai, P. Li and Y. Liang, "Characterization of Removable Elements with Respect to Having $k$ Disjoint Bases in a Matroid," Submitted.

[2] P. Li, Ph.D. Dissertation, West Virginia University, to be Completed in 2012.

[3] R. Haas, "Characterizations of Arboricity of Graphs," Ars Combinatoria, Vol. 63, 2002, pp. 129-137.

[4] D. Liu, H.-J. Lai and Z.-H. Chen, "Reinforcing the Number of Disjoint Spanning Trees," Ars Combinatoria, Vol. 93, 2009, pp. 113-127.

[5] D. J. A. Welsh, "Matroid Theory," Academic Press, London, New York, 1976.

[6] J. G. Oxley, "Matroid Theory," Oxford University Press, New York, 1992.

[7] J. A. Bondy and U. S. R. Murty, "Graph Theorym," Springer, New York, 2008.

[8] E. M. Palmer, "On the Spannig Tree Packing Number of a Graph, a Survey," Discrete Mathematics, Vol. 230, No. 1-3, 2001, pp. 13-21.

[9] C. St. J. A. Nash-Williams, "Edge-Disjoint Spanning Trees of Finite Graphs," Journal of the London Mathematical Society, Vol. 36, No. 1, 1961, pp. 445-450.

[10] W. T. Tutte, "On the Problem of Decomposing a Graph into $n$ Connected Factors," Journal of the London Mathematical Society, Vol. 36, No. 1, 1961, pp. 221-230.

[11] J. Edmonds, "Lehman's Switching Game and a Theorem of Tutte and Nash-Williams," Journal of Research of the National Bureau of Standards, Section B, Vol. 69B, 1965, pp. 73-77.

[12] C. St. J. A. Nash-Williams, "Decomposition of Fininte Graphs into Forest," Journal of the London Mathematical Society, Vol. 39, No. 1, 1964, p. 12.

[13] W. H. Cunningham, "Optimal Attack and Reinforcement of a Network," Journal of Associated Computer Machanism, Vol. 32, 1985, pp. 549-561.

[14] P. A. Catlin, J. W. Grossman, A. M. Hobbs and H.-J. Lai, "Fractional Arboricity, Strength and Principal Partitions in Graphs and Matroids," Discrete Applied Mathematics, Vol. 40, No. 1, 1992, pp. 285-302.

[15] A. M. Hobbs, "Computing Edge-Toughness and Fractional Arboricity," Contemporary Mathematics, Vol. 89 1989, pp. 89-106.

[16] A. M. Hobbs, L. Kannan, H.-J. Lai and H. Y. Lai, 
"Transforming a Graph into a 1-Balanced Graph," Discrete Applied Mathematics, Vol. 157, No. 1, 2009, pp. 300-308.

[17] A. M. Hobbs, L. Kannan, H.-J. Lai, H. Y. Lai and Q. W. Guo, "Balanced and 1-Balanced Graph Construction," Discrete Applied Mathematics, Accepted.

[18] P. A. Catlin, "Super-Eulerian Graphscollapsible Graphs, and Four-Cycles," Congressus Numerantium, Vol. 58, 1987, pp. 233-246.

[19] P. A. Catlin, Z. Han and H.-J. Lai, "Graphs without Spanning Closed Trails," Discrete Mathematics, Vol. 160,
No. 1-3, 1996, pp. 81-91.

[20] P. A. Catlin, "Super-Eulerian Graphs - A Survey," Journal of Graph Theory, Vol. 16, No. 2, 1992, pp. 177-196.

[21] Z. H. Chen and H.-J. Lai, "Reduction Techniques for Super-Eulerian Graphs and Related Topics - A Survey," Combinatorics and Graph Theory 95, Vol. 1, World Science Publishing, River Edge, New York, 1995.

[22] J. Bruno and L. Weinberg, "The Principal Minors of a Matroid," Linear Algebra and Its Applications, Vol. 4, 1971, pp. 17-54. 